project supported those who were lonely and at risk of isolation. A full review of our services took place following the closure of all hubs, reduction in staff and enforced shielding of our patients, including the impact of social interaction for those vulnerable and isolated.

Aims To enhance the patient experience by offering episodes of clinical care, supported with a range of therapy interventions. Patients, their carers, and those bereaved are then invited to attend informal social meet-ups, or take part in wellbeing activities at new social hubs in community spaces to foster compassion and peer support. The hubs maintain a connection to the hospice until the point where clinical care might once again be needed.

Methods

- Developed a blended approach of online and face-to-face assessments.

- Introduced defined episodes of therapeutic care $(6,8$ or 12 week groups).

- Patient-centred programmes focus on achieving specific personal goals (Fettes, Ashford, Maddox, 2018).

- All care led by Integrated Palliative Outcome Scale (IPOS)

- Therapy to Compassionate Community (Compassionate Communities UK) caseload transition.

- Set up new social hubs.

Results 53 patients transitioned (Apr 20-May 21). Increased feedback from 'Views on care' to aid evaluation. Carer experience significantly improved.

Conclusions We adapted our service to bring an exciting solution towards enhancing the patient and carer experience by replacing 'discharge' with 'episode of care' and offering a transition from clinical therapy to community caseload for continued support and connection. This model has also enhanced our family support service.

\section{P-92 COVID-19 PRESSURE BEDS: RELIEVING THE DEMAND ON ACUTE BEDS BY TRANSFERRING INTO HOSPICE CARE}

Karen Walker. Ashgate Hospicecare, Chesterfield, UK

\subsection{6/spcare-2021-Hospice.110}

Background Throughout the COVID-19 pandemic, health and care systems were under immense pressure. The hospice made the decision to assist the local trust and admit palliative patients who had tested positive for COVID-19 into six pressure beds. Building on the partnership between the hospice and local trust, the beds were accessed by the trust through a new patient pathway of admission between October 2020 and March 2021.

Aim To achieve a timely, safe and appropriate transfer of COVID-19 criteria led patients from primary to hospice care.

Methods A new admission transfer criteria was introduced patients with non-complex palliative care needs who were COVID-19 positive. A service level agreement was established to enable a consistent and collaborative approach. The ward sisters held daily morning meetings to discuss identified admissions. A designated wing was established to care for COVID19 positive patients to prevent transmission.

Results A total of 40 admissions were taken since Nov. 2020. The transfer of acute patients to hospice care resulted in 369 saved bed days. Families were allowed precious time during the last days of life for $65 \%$ of patients in a calm and caring environment maintaining privacy and dignity for patients in their last days. Able to facilitate safe discharges home when patients recovered from the virus. Despite the implemented patient criteria, all patients transferred still required medical input due to their complex needs.

Conclusion Our findings demonstrate that working in partnership with the wider health and care system improves patient access to hospice care. Invaluable patient and family experience provides strong evidence for the continuation of the additional six beds. Admission criteria must be robust and flexible. We can restart the pathway if a third wave occurs and the hospice is further exploring how we can continue to support our local trust with the option of flexible winter pressure beds.

\section{P-93 SETTING UP A 'VIRTUAL WARD' WHEN THE INPATIENT UNIT CLOSES: LEARNING FROM CRISIS AND OPPORTUNITY}

Arjun Kingdon, Matthew Sweeting, Eva Lew. Farleigh Hospice, Chelmsford, UK

\subsection{6/spcare-2021-Hospice.111}

Background Farleigh Hospice provides palliative and end-oflife care to the people of Mid Essex (population c. 400,000), with a dedicated inpatient unit (IPU) and community services. Problem The first wave of the COVID-19 pandemic catalysed organisational change with creation of 'Locality Care Teams' and the upskilling of non-clinical members of staff. Subsequently, the IPU closed and staff were reassigned to Locality Care Teams in November 2021, mobilising the workforce to care for more patients. However, there was a resulting potential for some patient and carer needs to go unmet.

Intervention A community-based nurse-led 'Virtual Ward' was created in mid-December at rapid pace, to support people to die at home. A detailed SOP was developed, specifying the purpose of the Virtual Ward, eligibility criteria, and referral, assessment and discharge processes. This information was disseminated to all teams. Data were collected throughout the time of IPU closure.

Outcome Within a short time, the virtual ward was well-utilised (mean 8.6 admissions per month). Referrals to the Virtual Ward were largely appropriate with mean length of stay seven days, median three days. $80 \%$ of patients admitted to the Virtual Ward died as expected while under its care; none were admitted to acute care services. Informal feedback has been positive from referrers, patients and family caregivers.

Learning The temporary closure of the IPU could have caused patient and carer needs to go unmet. Instead, the rapid set up of the Virtual Ward, alongside the reassignment of IPU staff and arrangements with other regional hospices, has enabled the local population's end-of-life care needs to be well met. During the pandemic the greatest need for palliative care services has been in the community (Etkind, Bone, Lovell, et al., 2020; Costantini, Sleeman, Peruselli, 2020). Farleigh Hospice's reconfiguration of services has proven to be effective and responsive. There is a need for more robust data collection to evaluate outcomes particularly with regard to patient and family caregiver feedback. 